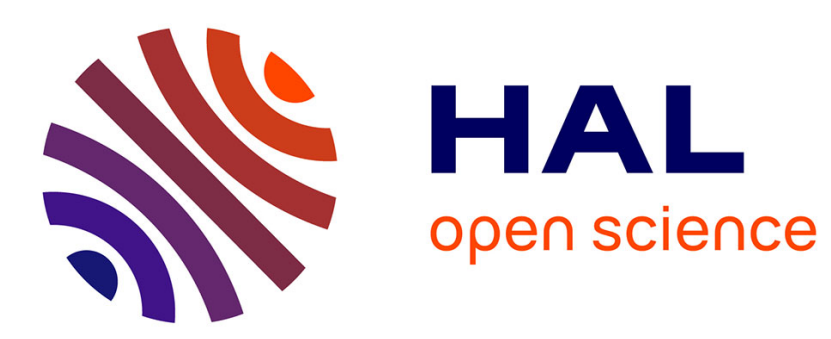

\title{
Mosaïque des intelligences dans l'organisation : Éléments de distinction entre hauts potentiels, talents et HQI.
}

Philippe Mouillot, Dominic Drillon, Nathalie Montargot

\section{To cite this version:}

Philippe Mouillot, Dominic Drillon, Nathalie Montargot. Mosaïque des intelligences dans l'organisation: Éléments de distinction entre hauts potentiels, talents et HQI.. Revue internationale de psychosociologie et de gestion des comportements organisationnels, 2018, XXIV (58), pp.171. 10.3917/rips1.058.0171 . hal-02123539

\section{HAL Id: hal-02123539 \\ https://hal.science/hal-02123539}

Submitted on 17 May 2019

HAL is a multi-disciplinary open access archive for the deposit and dissemination of scientific research documents, whether they are published or not. The documents may come from teaching and research institutions in France or abroad, or from public or private research centers.
L'archive ouverte pluridisciplinaire HAL, est destinée au dépôt et à la diffusion de documents scientifiques de niveau recherche, publiés ou non, émanant des établissements d'enseignement et de recherche français ou étrangers, des laboratoires publics ou privés. 


\section{MOSAÏQUE DES INTELLIGENCES DANS L'ORGANISATION : ÉLÉMENTS DE DISTINCTION ENTRE HAUTS POTENTIELS, TALENTS ET HQI \\ Philippe Mouillot, Dominic Drillon et Nathalie Montargot}

ESKA | «Revue internationale de psychosociologie et de gestion des comportements organisationnels »

2018/58 Vol. XXIV | pages 171 à 196

ISSN 2262-8401

ISBN 9782747228039

Article disponible en ligne à l'adresse :

https://www.cairn.info/revue-internationale-de-psychosociologie-de-gestion-descomportements-organisationnels-2018-58-page-171.htm

Distribution électronique Cairn.info pour ESKA.

(C) ESKA. Tous droits réservés pour tous pays.

La reproduction ou représentation de cet article, notamment par photocopie, n'est autorisée que dans les limites des conditions générales d'utilisation du site ou, le cas échéant, des conditions générales de la licence souscrite par votre établissement. Toute autre reproduction ou représentation, en tout ou partie, sous quelque forme et de quelque manière que ce soit, est interdite sauf accord préalable et écrit de l'éditeur, en dehors des cas prévus par la législation en vigueur en France. Il est précisé que son stockage dans une base de données est également interdit. 


\section{MOSAÏQUE}

\section{DES INTELLIGENCES DANS}

\section{L'ORGANISATION : \\ ÉLÉMENTS DE DISTINCTION ENTRE HAUTS POTENTIELS,}
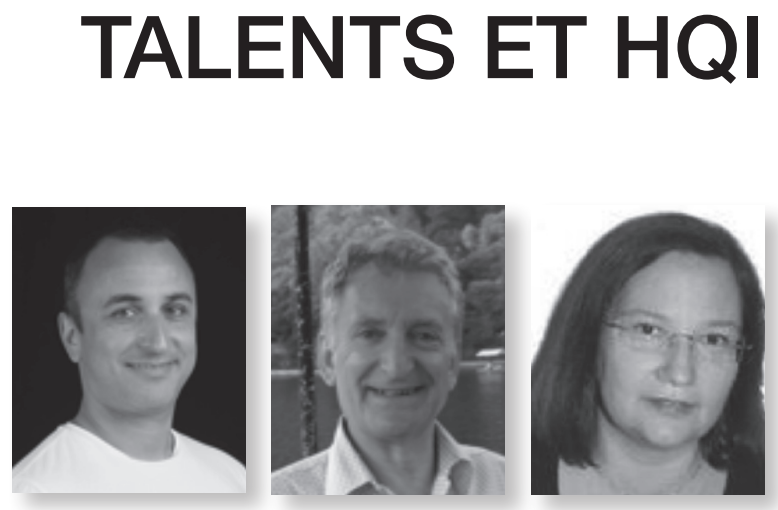

Philippe MOUILLOT ${ }^{109}$, Dominic DRILLON ${ }^{110}$ et Nathalie MONTARGOT ${ }^{111}$

109. Maître de Conférences HDR, Laboratoire CEREGE EA1722, IAE de Poitiers, 20 Rue Guillaume VII Le troubadour, 86000 Poitiers, pmouillot@poitiers.iae-france.fr

110. Enseignant-chercheur HDR, Laboratoire CEREGE EA1722, CRM La Rochelle Business School, 102 Rue de Coureilles, 17000 La Rochelle, drillond@esc-larochelle.fr

111. Enseignant-chercheur, Professeure Associée, Laboratoire CEREGE EA1722 CRM La Rochelle Business School, 102 Rue de Coureilles, 17000 La Rochelle, Chaire ESSEC du Changement, montargotn@esc-larochelle.fr 


\section{INTRODUCTION}

Les organisations évoluent dans un monde concurrentiel, où le capital intellectuel et humain s'avère être un atout considérable. Dans ce contexte, la fonction Ressources Humaines vise à les placer au cœur des processus de création de valeur. En effet, dans la mesure où ils ne sont ni capitalisés, ni partagés, leur déperdition entraine une perte de valeur considérable (Fourmy, 2013 ; Peretti, 2009 \& 2018).

Depuis près de vingt ans, la guerre des talents fait rage. Ces derniers sont en effet considérés comme des atouts incontournables dans un univers globalisé et fortement concurrentiel (Michaels, Handfield-Jones \& Axelrod, 2001 ; Minbaeva \& Collings, 2013). En 2015, le groupe Manpower a publié une étude portant sur la pénurie des talents ${ }^{112}$. Il ressort de celle-ci que plus de $38 \%$ des DRH recherchent le même type de profil. La rareté des candidats impacte négativement le fonctionnement des organisations, leurs activités et la satisfaction de leurs clients. En conséquence, les employeurs cherchent à développer des stratégies leur permettant d'attirer et de retenir les candidats de valeur. Les entreprises développent ainsi leur marque-employeur, des marques qui, à l'instar de Nestlé, Deloitte, l'Oréal ou encore Saint-Gobain, se positionnent comme accordant une grande importance aux valeurs véhiculées par leur organisation, au pouvoir d'attractivité des candidatures et à la gestion de carrière des collaborateurs. Ces derniers présentent d'ailleurs des profils souvent similaires, non exempts de phénomènes de reproduction sociale (Bourdieu, 1980). Ils sont souvent diplômés d'écoles prestigieuses, jeunes et mobiles, performants dans leurs missions et présentent un fort potentiel d'évolution. Ces individus suivent alors des programmes spécifiques de développement et de fidélisation, leur permettant d'accéder aux plus hautes fonctions dans l'organisation, qu'ils soient ou non informés de leur participation à de tels programmes.

Nonobstant les efforts réalisés par certaines firmes dans la gestion des talents, la détection du niveau et les formes d'intelligence restent rarement mesurée dans les processus de sélection (MARTIN, 2018). En effet, les organisations préfèrent miser sur des indicateurs connus et reconnus, tels que les diplômes ou l'expérience capitalisée (Bourdieu, 1980 ; MintZBERG, 2005). Or l'intelligence, tout particulièrement dans un contexte turbulent où l'agilité organisationnelle est requise, constitue un atout favorisant une création de valeur stable et pérenne. Pourtant, nous voyons bien que moult environnements professionnels sont sensibles à la notion d'intelligence au point de mettre en place des tentatives de détection et d'accompagnement du capital humain. De ce paradoxe, nous sommes arrivés à la conclusion que l'intelligence était considérée, peut-être même finalement mesurée, mais dans une contextualisation différente,

112. http://www.manpowergroup.fr/penuriedetalents2015/ Non repris en bibliographie 
notamment sémantique. Et à ce titre, nous nous sommes donc demandé si la volonté des managers consistant à considérer l'intelligence ne se diluait pas dans celle de gérer les talents et les hauts potentiels, trois notions fréquemment substituées les unes aux autres malgré l'existence de différences marquées, d'où notre problématique : les DRH gèrent-ils déjà les HQI sans le savoir, les gèrent-ils en les nommant alternativement Hauts Potentiels ou encore Talents, ou bien ne les gèrent-ils pas par méconnaissance du phénomène?

Nous pensons que la clarification des éléments qui distinguent les HQI des talents et des hauts potentiels est une étape cruciale à la mise en place de solutions managériales permettant leur gestion, car les uns et les autres ne soulèvent pas les mêmes questions organisationnelles. Pour clairement distinguer ces trois concepts que les praticiens substituent trop souvent, cet article a donc pour objectif d'explorer la littérature afin de délimiter les champs respectifs du talent, du potentiel et du HQI. Nous verrons notamment s'il existe des glissements sémantiques entre la littérature francophone et anglophone, donnant ainsi un fondement épistémologique compréhensiviste à notre recherche.

\section{INTELLIGENCE, OU PLUTOT INTELLIGENCES}

L'intelligence fait sans doute partie des concepts les plus complexes à mesurer efficacement. Car même en la déconnectant de son pendant émotionnel, ce que nous ferons dans cette recherche, car les variables émotionnelles sont délicates à circonscrire, l'intelligence reste bien mystérieuse à de nombreux égards. Par exemple, difficile d'observer des constantes intellectuelles ; un sujet considéré comme très intelligent peut échouer dans la résolution d'un problème simple sans que nous soyons réellement capables d'en comprendre les raisons. La complexité du cerveau et notre connaissance limitée de son fonctionnement expliquent sans doute ces lacunes. Aussi, vouloir mesurer l'intelligence fait irrémédiablement appel à des outils fiables mais seulement capables de mesurer un nombre limité de traits intellectuels. Comme le souligne WeChSLER (1944), un outil servant à mesurer l'intelligence ne peut donner qu'un indicateur de capacité globale d'une personne, capacité elle-même fondée à partir de l'agrégation de plusieurs variables, parfois très volatiles.

A ce titre, les tests d'intelligence ne peuvent donc pas mesurer tout de l'intelligence. Cette dernière n'existe donc que dans son fonctionnement et ne se prête peut-être pas à une analyse détaillée, sans parler des problèmes d'interprétation des résultats d'un test d'intelligence. C'est sans doute la raison pour laquelle une définition consensuelle et rigide de l'intelligence n'a jamais réussi à s'imposer. Le Quotient Intellectuel, indicateur relativement universel de la mesure de l'intelligence, s'évalue actuellement 
sur la base d'un écart à la moyenne ou de l'appartenance à une minorité statistique en termes de réussite à des tests. Cette acception est, entre autres, celle de la MENSA ${ }^{113}$. Dès lors, cela a pour conséquence fâcheuse de permettre le classement des individus et de faire rentrer l'intelligence dans une échelle de valeur comme le seraient la beauté ou le bonheur. Les adversaires des tests et du déterminisme biologique de l'intelligence (Gould, 1981 ; SAMELSON, 1982) avancent aussi l'idée que des facteurs extérieurs influent sur la réussite d'un test, tel l'aspect culturel du contenu, le niveau de préparation de la personne testée ou les conditions de passation. Parallèlement, à la suite des travaux de Gardner (1983, 1993 \& 1999), on ne parlera plus d'unicité de l'intelligence mais d'intelligences multiples en ce sens que l'intelligence serait finalement composée de plusieurs variables (MARTIN, 2018).

L'intelligence semble donc être un concept composé de multiples concordances, aucune ne réussissant finalement à clairement distinguer l'humain intelligent de celui qui ne le serait pas. L'approche aristotélicienne que l'homme est un animal doué de raison n'aide d'ailleurs pas à circonscrire la notion d'intelligence. En effet, la raison suggère la présence d'une certaine forme de sagesse, raison et sagesse étant difficiles à désolidariser d'une certaine intelligence.

Le débat philosophique semble donc infini et définir l'intelligence comme la mesurer peut sembler inutile, voire impossible. Mais il n'en demeure pas moins que si l'on revient au domaine de l'organisation, de multiples tâches existent qui doivent être réalisées, des tâches effectuées par des personnes différentes et dont certaines seraient en mesure d'en réaliser une majorité là où d'autres ne le seraient pas, le tout avec des degrés très variés de performance.

Cela nous invite donc sans doute à entamer notre réflexion en traitant de la notion de compétence. Et avant même d'étendre son acception à la psychologie, cette dernière est déjà loin de faire l'unanimité dans l'organisation puisque gestionnaires, économistes et sociologues n'arrivent pas à s'accorder sur une acception commune de la compétence (Mouillot et al., 2000). En 1973, David McClelland était relativement précurseur en la matière en montrant la complexité de la compétence, cette dernière étant, selon lui, trop souvent calquée sur des exemples ou sur les besoins des organisations, alors que l'identification de compétences naturelles individuelles aurait sans doute été bien plus profitable en termes de variété de création de valeur. D'autres auteurs ont même essayé de modéliser la compétence (SPEncer \& SPEncer, 1993 ; Chouhan \& Srivastava, 2014) pour faire face, notamment, aux exigences des économies de la connaissance (Mouillot, 2013). Ces auteurs soutiennent par-

113. https://mensa-france.net/ : association internationale officiellement habilitée à diagnostiquer le HQI et à en gérer les conséquences. 
ticulièrement le recours aux compétences plutôt qu'aux techniques financières, marketing ou encore stratégiques dans les choix d'investissements susceptibles de développer les avantages concurrentiels des organisations. A partir des travaux de McClelland, Richard Boyatzis a soutenu en 2008 que les compétences professionnelles ne devaient pas seulement être identifiées mais créées, notamment grâce à la mise en œuvre de programmes de formation. En d'autres termes, BoyATZIs ne suggérait pas de trouver la compétence en fonction d'un besoin identifié en amont mais de la créer. D'ailleurs, de célèbres entreprises telles que AccorHotels, L'Oréal, Manpower, Google, Apple ou encore Facebook ont rapidement créé leurs propres académies.

Comme le souligne Perrenoud (2018) la notion de compétences est polysémique dans la mesure où elle permet d'agir efficacement dans un type défini de situation en s'appuyant sur des connaissances sans s'y réduire. Les compétences ne sont jamais la simple mise en œuvre objective et rationnelle de procédures, de modèles ou de connaissances. L'intelligence est peut-être ce catalyseur capable de mixer et de métaboliser des connaissances, de l'expérience, de l'intuition. En 2004, Le Boterf apportait une distinction importante entre être compétent et avoir des compétences. Pour le premier, c'est être capable d'agir et de réussir dans différentes situations. Pour le second, il s'agit de disposer de ressources pour affronter un environnement donné avec efficacité ou efficience. Les Organisations recherchent donc davantage des personnes qui agissent avec compétence et pas uniquement des employés compétents. Ces divers questionnements sur la nature et le rôle de la fonction RH sont sans doute à l'origine d'une refonte de la réflexion qui a ainsi muté de l'observation de bonnes pratiques à leur audit. Cette remise en cause a débuté dès 1992, notamment avec Peter Boxall même si d'autres auteurs avaient tiré la sonnette d'alarme dès 1978 (LEGGE, 1978 ; Dimick \& Murray, 1978 ; Mahoney \& Deckop, 1986 ; Palmer, 1988 ; Sisson, 1993 ; Truss \& Gratton, 1994 ; Malik, 2009). Ces critiques mettaient déjà en exergue le fait que l'employeur aurait un rôle de plus en plus prégnant dans la gestion de ses ressources humaines (IRwIN, 2008). Depuis 1989, notamment avec Karen LegGe, la fonction RH est donc complètement intégrée à la performance de l'organisation en termes de causalités.

Dans la lignée des travaux de McClelland (1973), de Legge (1978 \& 1989) ou de Boyatzis (2008), précurseurs en termes de critique de la fonction RH, les compétences sont considérées au niveau de l'individu créateur de valeur. On constate que ces approches ne discutent ni les modes d'acquisition de ces compétences, ni les facultés résilientes des individus à adapter leurs compétences face à des environnements nouveaux ou des problématiques nouvelles. Ces auteurs axent leur réflexion sur la raison d'être de la fonction $\mathrm{RH}$, notamment sur le rôle et la responsabilité du manager et de l'organisation à fournir aux employés des environ- 
nements d'apprentissage dans lesquels l'acquisition des compétences serait facilitée. Soit ; mais cela revient à questionner, en amont, les capacités intellectuelles que les employés sont en mesure de mobiliser pour acquérir ces compétences. Et force est de constater que plus le candidat sera intellectuellement flexible et performant, plus il aura accès à des postes aux responsabilités importantes. On en revient peut-être ici à l'approche darwiniste de la faculté d'adaptation des espèces mais, finalement, dans un monde dans lequel il est de plus en plus rare de faire toute une carrière dans la même organisation et aux mêmes fonctions, la capacité d'acquérir rapidement des compétences nouvelles ou bien d'adapter des compétences déjà maîtrisées reste la condition sine qua non de l'évolution professionnelle. Par conséquent, il nous semble important de traiter la notion d'intelligence individuelle dans l'entreprise comme étant l'un des ancrages fondamentaux à l'acquisition et au développement de la compétence, ainsi qu'à la remise en cause des modèles de gestion des ressources humaines. Car tout le monde ne peut pas tout faire et il est de la responsabilité des managers d'identifier les potentiels comme les limites des uns et des autres.

Et c'est à ce stade que nous touchons le cœur de notre réflexion. En effet, le DRH devra distinguer l'employé capable d'acquérir de nouvelles compétences - ou de transposer des compétences existantes - de celui qui n'y arrive pas. Bien entendu, la volonté, les encouragements et la qualité de l'environnement professionnel jouent un rôle dans ces dynamiques. Malgré tout, quand bien même toutes les conditions du bien-être au travail seraient réunies et quand bien même nous serions dans un cadre clinique dans lequel nous pourrions évaluer deux candidats aux volontés équivalentes de développement personnel, il n'en demeure pas moins que l'intelligence fondamentale resterait une variable discriminante du potentiel de l'un face à l'autre. Pour autant, ce potentiel détecté chez certains employés est-il un facteur qui est directement en lien avec des capacités intellectuelles ? Et dans cette même logique, la talent est-il synonyme d'intelligence, de compétence, des deux ou bien est-il la conséquence d'autres éléments?

Ces questions sont importantes car nous pensons que si les managers n'utilisent pas les bonnes terminologies, les modèles RH et la nature des investissements internes pourraient en être affectés.

Maintenant que nous avons montré qu'en amont de toute fabrication ou évolution d'une compétence, il y a un terrain qui est en lien avec l'intelligence (i.e. profondeur de compréhension, vitesse d'acquisition, résilience), le moment est venu de discuter ce qui réunit comme ce qui distingue les talents, les hauts potentiels et les HQI afin de fournir aux managers un cadre conceptuel et sémantique leur permettant d'affiner leur gestion des ressources humaines. 


\section{DE LA QUESTION DES TALENTS}

Jusqu'alors réservée aux domaines sportifs ou artistiques, la notion de talent professionnel combine intrinsèquement une dimension organisationnelle et individuelle (Besseyre des Horts, 2011). Elle s'est développée dans les années 2000, suite à la parution d'un ouvrage rédigé par des consultants du Cabinet Mc KinsEY, faisant référence à « la guerre des talents » (Michaels, HandField-Jones \& Axelrod, 2001). Les caractéristiques sociodémographiques de l'époque, caractérisées par l'imposante cohorte des baby-boomers remplacée par des générations moins nombreuses, la mondialisation, l'hyper compétition entre les firmes, leurs risques et manque de visibilité dans un contexte de crises et d'incertitudes, expliquent la course aux talents dans les organisations (Minbaeva \& Collings, 2013). Cette guerre est représentative d'une logique de marché dans laquelle les talents sont perçus comme des actifs économiques indispensables (AL Ariss et al., 2014).

Si les définitions du terme talent font l'objet de recherches (Thunnissen et al., 2013), celles-ci peinent cependant à stabiliser scientifiquement la notion, tant elle est polysémique (Mirallès, 2007 ; Vaiman \& Collings, 2013 ; Dries, 2013 ; Al Ariss et al. ; 2014). La notion de talent n'est d'ailleurs appliquée que de façon récente à l'entreprise. Maurice Thévenet (2012, p. 254) estime également qu'elle peut « recouvrir des notions qui ne sont pas toutes compatibles entre elles ». Elle continue ainsi à être mise en perspective avec les concepts traditionnels de la GRH : la compétence, la qualification, l'expertise, le potentiel et le haut potentiel. Pierre Mirallès (2007) met le talent en équation. Pour lui, Talent = Excellence + Différence. Il l'applique dans sa proposition de modèle de management des talents, en l'opposant « presque terme à terme au modèle de la compétence, comme à celui des qualifications » (Mirallès, 2007, p. 40). En effet, le talent, contrairement à la compétence, correspond à une supériorité individuelle dans un domaine précis et à un moment donné. Le talent se rapporte à une combinaison subtile de compétences rares, provenant d'un individu hors-norme dont les compétences exceptionnelles ne figurent pas sur les référentiels métiers préétablis par les entreprises (ThÉvenet \& Dejoux, 2010). Il repose sur un triptyque, alliant compétence, performance et leadership (Ulrich \& SMALlwood, 2012).

L'ANDRH (Association Nationale des DRH) a, pour sa part, publié en 2017 une nouvelle édition de son étude annuelle sur la gestion des talents dans les entreprises françaises ${ }^{114}$. Elle identifie cinq dimensions individuelles : la capacité et la volonté à évoluer : l'engagement, la capacité à occuper des postes de direction, le sens des responsabilités et la performance élevée. Ces dimensions invitent les firmes

114. http://www.andrh.fr/l-actualite/liste-des-actualites/la-gestion-des-talents-dans-les-entreprises-francaises-2017 Non repris en bibliographie 
à identifier et faire fructifier leur investissement dans le capital humain, afin de trouver parmi ses membres les éléments les plus susceptibles de contribuer à leur performance. Le talent individuel promis à une capacité d'évolution élevée, doit permettre de faire la différence et de bénéficier d'un avantage concurrentiel durable (Mirallès, 2007).

Les processus organisationnels relatifs à son management suscitent l'intérêt des chercheurs (CAPPelli \& KelleR, 2014 ; NAUlleAU, 2015). Ils étudient tout particulièrement les politiques et pratiques d'attraction, de développement et de rétention des talents (Boudreau \& Ramstad, 2005), qu'il est important de mettre en lien avec les objectifs stratégiques, la culture organisationnelle et les normes organisationnelles (GARROW \& HiRSCH, 2008). Les résultats attendus par le management des talents portent, sur un plan individuel, sur un renforcement de la motivation, de l'engagement, de la confiance, du bien-être, et sur un plan collectif, sur la performance et la compétitivité organisationnelle (Collings \& Mellahi, 2009).

Le talent professionnel est-il inné ou acquis par le travail ? Sur ce point, les courants philosophiques mais également académiques s'opposent. Le talent serait inné et, de fait, selon KANT (1790) ou DuRKHEIM (1893) en tout cas, inégalitaire par essence. A l'opposé, selon NieTzChe (1878), il serait « acquis grâce à une forte volonté de développement et à une importante capacité de travail » (JANAND et al., 2016, p. 46). Ce débat philosophie imprègne encore les approches de la littérature. Le talent est en effet souvent considéré comme un don naturel, des capacités remarquables, voire géniales, et faisant partie du seul capital héréditaire (NAQVI, 2009). Il appartiendrait cependant à l'individu de préserver et de mettre en valeur ce capital lors de son parcours professionnel. Ce don naturel demeure indissociable des compétences, connaissances et expériences acquises, ainsi que de la personnalité, l'énergie et la capacité individuelle à apprendre et à évoluer (Michaels, HANDFIELD-Jones \& AXELROD, 2001 ; BuCKINGHAM \& Vosburgh, 2001). Si le talent semble idiosyncrasique, son expression nécessite cependant la mise en œuvre de processus organisationnels spécifiques, afin d'en tirer une valeur économique. « D'où une double dépendance : des talents par rapport aux organisations capables de leur offrir les meilleures conditions d'expression ; des organisations par rapport aux talents capables de leur permettre de viser l'excellence dans leur sphère d'activité » (Mirallès, 2007, p. 38).

Sur le plan de savoir qui sont réellement les talents dans les organisations, trois acceptions dominent.

La première, exclusive, se réfère à des individus disposant de compétences rares, nous l'avons vu. Une élite composée d'individus très recherchés sur le marché produit des résultats supérieurs à ceux de ses pairs. 
Une deuxième acception inclusive peut être relevée. Les talents désignent alors non un petit nombre d'individus, mais au contraire l'ensemble des collaborateurs dont le potentiel individuel a pu ou non être révélé (Besseyre des HorTs, 2011 ; Gallardo-Gallardo et al., 2012). Dans cette acception, des talents jusqu'alors inexploités ou non révélés, pourraient, grâce à des leviers, révéler leur plein potentiel (Peretti, 2009). L'organisation a donc tout intérêt à rechercher les meilleures conditions de son expression et de sa valorisation. La gestion des talents doit en conséquence se fonder sur des bonnes pratiques, mais aussi s'aligner sur la stratégie et la culture organisationnelle, ses règles et ses processus (GARROW \& HiRSCH, 2008).

Une dernière approche fait référence de manière plus indirecte à l'individu. C'est le poste et sa position dans l'organisation qui deviennent centraux (DeJoux, 2014). En effet, certaines personnes-clés disposent d'atouts personnels exceptionnels et contrôlent des processus déterminants pour l'organisation (Mirallès, 2007). Dans ce dernier cas, certains métiers et fonctions stratégiques sont valorisés par l'organisation (Boudreau et Ramstad, 2005 ; Collings et Mellahi, 2009). Les individus occupant ces fonctions sont alors assimilés à de « hauts potentiels 》 (BESSEYRE DES HORTS, 2011). La valeur économique d'un talent reste relative à un contexte ou à un domaine (Besseyre des Horts, 2011 ; Gallardo-Gallardo et al., 2012). Elle s'apprécie également à un moment précis, au moyen de comparaisons et classements de leurs performances actuelles et futures. Naulleau (2015) relève à ce sujet un paradoxe dans la littérature : il pointe une inconsistance théorique de la notion de talent avec des définitions polysémiques non stabilisées et des divergences d'interprétation entre les acteurs tout en voulant objectiver une démarche de management des talents, à l'aide de modèles d'aide à la décision et de pratiques normalisantes.

Que l'on se place dans la vision d'Emmanuel Kant et d'Émile Durkheim ou bien dans celle de Friedrich NiETzChe, l'intelligence reste un facteur commun aux deux acceptions : dans le premier cas, elle est innée, dans le second elle permet l'acquisition de compétences grâce, notamment, à des capacités de métabolisation et de travail qui sont très supérieures à la moyenne. Même si le talent est un don naturel, l'individu talentueux reste seul maître à bord quant à sa volonté de le développer par le travail, de le remettre en question puis de le confronter à de nouvelles situations tout au long de sa vie, notamment professionnelle. Le talent est donc créateur et développeur de compétences. A ce titre, il est source de potentiel. Mais il reste néanmoins lié à une ou plusieurs formes d'intelligence telles que définies par Howard GARDNER en 1983. Cette question de la volonté de ce que l'on souhaite faire d'un outil est donc à la source de la réflexion sur le potentiel, concept que nous allons maintenant explorer. 


\section{DE LA QUESTION DES HAUTS POTENTIELS}

La notion de haut potentiel est extrêmement complexe à définir car la littérature ne s'accorde pas sur une acception définitive. En effet, le potentiel peut être lié aux capacités intellectuelles d'un individu, à sa volonté de progresser, aux réseaux auxquels il appartient, à ses appétences professionnelles, voire aux quatre en même temps. La notion de potentiel humain est donc difficile à circonscrire car elle peut être un amalgame de plusieurs variables qui, finalement, ne remplissent qu'une fonction prédictive. En outre, un potentiel, qui plus est « haut», n'est pas automatiquement assuré d'atteindre des objectifs exceptionnels. Il est donc plus ici question de promesse sur un avenir incertain que de mesure précise permettant d'investir du temps et de l'argent sur un individu.

D'une manière générale, la littérature semble accorder du crédit au fait que le potentiel ne peut se développer que dans un environnement de confiance (MALIK \& Singh, 2014). En effet, le côté anxiogène de l'exigence d'une performance est relativement compensé par le fait que si les dirigeants ont confiance dans un individu, celui-ci développera naturellement des signes indiquant la présence d'un potentiel. Les auteurs restent tout de même prudents car depuis 2009 et les travaux de GARDNER \& WRIGHT qui questionnent les multitudes de publications sur les liens pouvant exister entre les pratiques RH et les réussites des entreprises, les auteurs se sont aperçus que les réponses des personnes interrogées pouvaient être biaisées par les résultats des organisations justement. En d'autres termes, le succès organisationnel servirait de point d'ancrage à la qualité de la GRH alors que, finalement, les liens ne seraient pas si évidents que cela. Nous restons donc dans cette perspective de la difficulté à clairement considérer un « potentiel » en termes de critères fixes. D'autres auteurs (LANG, 2012) questionnent même la distinction entre le potentiel et la potentialité. Ici, le potentiel concerne le domaine du possible par opposition à celui de l'actualité, réalité déjà révélée. Quant à la potentialité, il s'agirait de la puissance latente de transformer la réflexion en action. Il s'agit alors là d'un domaine important puisqu'en plus de la capacité à la fois physique, intellectuelle, voire organisationnelle, les auteurs rajoutent l'élément de volonté. On rejoint ici la question du talent et de la volonté d'utiliser des compétences innées et de développer des compétences acquises.

La notion de potentiel a surtout été étudiée en GRH sous l'angle des " hauts potentiels », ces derniers étant traditionnellement identifiés parmi des cadres supérieurs, souvent expatriés, issus de viviers de « jeunes talents » destinés à prendre des responsabilités. A nouveau, le « potentiel » se confond donc ici avec le « talent». Il n'en demeure pas moins un pari virtuel sur l'avenir, une promesse de réalisation personnelle et professionnelle qu'un dirigeant « extrait» d'un employé. Le potentiel est complexe (CHAPELIER et al., 1999) dans la mesure où il métabolise des éléments organisationnels (i.e. possibilités de croissance 
en interne), des éléments personnels (i.e. degré de confiance accordé au collaborateur), des éléments temporels (i.e. succès et échecs passés), des éléments privés (i.e. influence et soutien de la famille) et des éléments psychologiques (i.e. volonté, résilience). De ce point de vue, le potentiel est d'ailleurs souvent directement associé aux deux domaines de la GRH que sont le recrutement et la rémunération, donc, par extension, encore une fois à la gestion des talents. Cette dernière repose sur la segmentation des RH recommandée par la Resource-Based View et la théorie du capital humain de l'économiste Gary BECKER (LePAK \& SNeLL, 1999) dont la pensée suggère qu'il est possible d'évaluer les variables économiques qui influencent, même marginalement, les comportements humains. Becker travaille ainsi à partir du postulat selon lequel il existe une rationalité économique pour chaque action de la vie quotidienne, notamment sociale.

La confiance entre le dirigeant et l'employé considéré comme ayant un haut potentiel est donc primordiale pour donner naissance à une réelle réalisation de soi puisque si l'entreprise ne peut pas maîtriser la totalité des variables qui composent la perception du potentiel, elle peut les connaître, ne serait-ce qu'au cours d'entretiens annuels ou d'échanges concernant des perspectives de progression interne. La perception du potentiel reste de toute façon subjective car les résultats passés peuvent ou non présager de l'avenir ; en revanche, confiance ne signifie pas surprotection, ce dont le dirigeant doit se préserver (Mirallès, 2007). En outre, si un potentiel constitue un faisceau de possibilités, encore faut-il savoir à quoi il peut servir. Car sans objectif ni calibrage du chemin d'évolution, un potentiel peut rapidement devenir une charge (RoussiLLon, 2006). Le pari du potentiel d'un employé est donc certainement aussi risqué que celui de partager le diagnostic du dirigeant avec la personne ciblée. En effet, supputer la présence d'un potentiel chez un collaborateur et ne pas le lui dire, c'est passer à côté d'une opportunité de motivation (incentive). En revanche, exposer ses espoirs, c'est prendre le risque d'apparition d'une dissonance cognitive chez le collaborateur entre le message reçu et les actions finalement réalisées. Par exemple, ce collaborateur pourra soit tout mettre en œuvre pour atteindre les objectifs fixés et ne pas décevoir son $\mathrm{n}+1$, soit attendre que les choses se fassent naturellement. L'annonce d'un potentiel peut donc avoir des répercussions actives comme passives chez le personnel ciblé (Roger \& TREMblay, 1992).

Dernière difficulté, la perception des résultats de la part du management (FESSER \& Pellissier-Tanon, 2007). Car plus la perception de la présence d'un haut potentiel est forte, moins les résultats seront valorisés. En effet, un haut potentiel qui obtient des résultats supérieurs à la moyenne ne se trouve finalement que dans sa propre moyenne, ce qui ne déclenche pas de félicitations particulières. Être identifié comme étant un haut potentiel est donc positif en termes de déblocage de ressources pour que ce dernier puisse accomplir pour s'accomplir, mais négatif en termes de résultats car les attentes sont si hautes que les résultats sont rarement supérieurs aux objectifs. 
Il en résulte que la gestion par le potentiel est donc une fausse bonne chose, menant souvent les uns et les autres, recruteurs comme hauts potentiels, à finalement rester à leur place, la perception et les actions liées au potentiel étant discrètes, non formellement liées, voire simplement subconscientes. Christophe FALCOZ (2001) va même plus loin en défendant l'idée selon laquelle les grands dirigeants ne savent jamais qu'ils ont été eux-mêmes choisis.

Franck Bournois et Jean-Luc Cerdin (ScouARneC, 2010) ont travaillé sur ces catégories de population, employant alternativement le terme de potentiel ou de talent, ces derniers étant d'acception similaire pour ces auteurs. Véronique DE SAINT-GinIEZ (2000) a aussi travaillé sur la notion de haut potentiel mais selon une approche plus critique. L'auteure s'attache à montrer que l'on détecte le potentiel à partir de signes perçus comme positifs - un diplôme par exemple - et identifie le potentiel comme un construit accepté et valorisé socialement mais aussi discutable. A ce titre, en psychologie sociale, Claude LÉvy-Leboyer (2009) a beaucoup travaillé sur l'évaluation des compétences. Pour elle, haut potentiel rime avec aptitudes. Quant à la méthode des habiletés (LEMOINE, 2003) consistant à recruter sur la base des habiletés identifiées chez des candidats, elle opérationnalise la détection du potentiel mais cette fois dans le cadre d'applications à des métiers d'exécution, le plus souvent manuels.

Le construit AMO (Ability - Motivation - Opportunity) offre également des pistes intéressantes d'exploration du potentiel dans la mesure où il considère que la performance individuelle est une synthèse d'habileté, de motivation et d'opportunités (Boxall \& Purcell, 2003 ; Marin-Garcia \& Tomas, 2016).

Le potentiel semble donc être un habile mélange d'intelligence, de degré d'intégration au sein d'une structure dans laquelle de la confiance est échangée et de volonté de réussite, tout au moins de volonté d'action, le tout dans un environnement rendu propice à l'éclosion de bonnes pratiques. Mais il reste un pari sur l'évolution d'une personne, cette évolution pouvant être la conséquence de nombreuses variables intrinsèques et extrinsèques. La distinction entre potentiel et haut potentiel ne vient finalement que de l'amplitude de performance attendue et/ou de la durée espérée de cette performance. Nonobstant ces éléments, le potentiel d'un individu reste complètement aléatoire, aucune grille, présence d'items ou méthodologie ne permettant de clairement identifier une personne à potentiel ou haut potentiel avec exactitude, ni présager de son évolution et de ses résultats, que celle-ci soit informée ou non des espoirs qui pèsent sur elle.

A partir de ces éléments, il nous semble donc raisonnable de penser que si le potentiel est un pari sur l'avenir, la cote de ce pari est éminemment fonction de la puissance intellectuelle du sujet, puissance intellectuelle agrégeant les notions de compétences 
ou de talents, de capacité de travail, de diversité de la réflexion, de profondeur de l'analyse, de vitesse d'exécution et de résilience face à l'inconnu. Autrement dit, que l'on choisisse de se placer dans un paradigme de talent ou de potentiel, le HQI apparaît comme étant la seule variable qui soit commune à l'ensemble, surtout si l'on considère l'idée selon laquelle il n'existe pas une intelligence mais plusieurs, ce que nous allons développer maintenant.

\section{DE LA QUESTION DES HQI}

Comme dans le cadre du haut potentiel, l'identification des individus qualifiés de « HQI » est une tâche délicate (Guignard \& ZeNASNI, 2004). La principale difficulté rencontrée lorsque l'on s'intéresse à ces populations vient de l'absence d'une définition unique ou consensuelle de ce concept. La littérature anglophone utilise le terme générique de « gifted » (doué(e)) alors que bien souvent, nous parlons en France d'individus à « haut potentiel», sans parler des enfants que l'on qualifiera de « précoces », une absurdité puisque l'intelligence est une mécanique stable. Les enfants HQI ne font donc pas les choses plus tôt, ils les font tout simplement plus vite et mieux.

L'utilisation du terme de « haut potentiel » pour qualifier une personne dotée d'un QI élevé engendrerait encore plus de confusion avec la définition couramment admise pour lesdits hauts potentiels dans les organisations, nous l'avons vu, d'autant que le terme « doué » se décline facilement.

Apparu pour la première fois dans la littérature académique chez de AJURIAGUERRA (1959), ce néologisme sera repris quelques années plus tard par des chercheurs québécois. Il est depuis largement utilisé dans d'autres pays francophones. Il signifie qu'un individu "surdoué », enfant ou adulte, dispose d'une intelligence supérieure à la moyenne. Mais le titre de "surdoué » est souvent récusé, particulièrement par ceux qui sont identifiés comme tels. Les termes « douance» ou « surdouance» sont également discutés par les scientifiques car ils peuvent laisser supposer qu'ils se réfèrent à un don particulier, donc qu'ils ont une origine héréditaire ou génétique, en l'occurrence « talentueuse ». D'autres, au contraire, vont privilégier l'influence environnementale ou culturelle. Nait-on intelligent ou le devient-on ? Sur ces questions, la science n'a pas encore tranché entre les partisans de l'influence génétique et ceux qui défendent le rôle de facteurs facilitants comme l'environnement ou l'éducation. Les critiques sur les recherches portant sur l'héritabilité ou non de l'intelligence sont nombreuses et parfois virulentes, particulièrement par l'utilisation qui pourrait en être faite.

Ellen WinNER, chercheur en psychologie à l'université de Harvard, adopte un juste milieu original (1997). Elle prétend que la douance est un mélange de nature et de 
culture, mais que cette douance n'est pas valorisée par crainte d'élitisme malgré le fait qu'elle représente une source potentielle de création de valeur pour les organisations qui sauraient détecter les personnes douées, les accompagner et leur fournir un environnement adapté, le tout en estimant qu'il serait prétentieux d'affirmer connaitre l'avenir d'un enfant « surdoué ». L'auteur recense quelques caractéristiques des futurs génies : leur indépendance, leur capacité à remettre en cause les pratiques, les habitudes, etc. GARDNER, (1983, 1993 \& 1999) considère que l'intelligence est utile et nécessaire pour résoudre des problèmes et traiter de l'information ; elle est ainsi créative, et ce quel que soit le contexte et l'environnement culturel. Le rôle de l'intelligence est donc capital, en effet, sans l'intelligence, impossible d'apprendre, de se rappeler ou de traiter efficacement des informations (GLYNN, 1996).

Nous voyons donc que l'intelligence fait partie de ces sujets sur lesquels nous avons tous une opinion sans être vraiment en mesure de nous accorder, un peu à l'instar de ce que Paul Watzlawick (1964) avait souligné au sujet de la communication il y a plus de cinquante ans lorsqu'il avait noté que malgré notre capacité universelle à communiquer, nous n'étions pas en mesure de communiquer sur la communication. Nous sommes tous doués d'intelligence mais sans avoir l'intelligence d'être capable de la définir et de la mesurer universellement. Certes, la psychologie n'est pas en reste en termes d'efforts pour y parvenir, la multiplicité des tests le prouve, encore à l'heure actuelle lorsque les psychologues utilisent des protocoles précis pour mesurer l'intelligence. En ce qui concerne notre acception de cette dernière, nous nous inscrivons dans la lignée de Jean Piaget (1936) pour qui l'intelligence n'est pas ce que l'on sait faire mais ce que l'on fait concrètement quand on ne sait a priori pas faire, et plus récemment dans celle de NisBetT et al. (2012) qui définissent l'intelligence comme la faculté de comprendre, de s'adapter, et de découvrir des relations entre des faits, voire même d'imaginer des solutions pour résoudre des problèmes rencontrés pour la première fois.

Mais être capable de trouver une solution à des situations rencontrées pour la première fois, forçant ainsi l'individu à être lui-même acteur et créateur de nouveauté ne résout pas la question de la nature de la solution, ni du chemin cognitif emprunté pour y parvenir. Le premier à s'être penché sur la question est HowARD GARDNER dès 1983. Il a défini les huit formes d'intelligence qui façonnent l'intelligence générale (SpeArman, 1904). Il s'agit des intelligences verbale (i.e. capacité à exprimer des idées de façon claire et intelligible du plus grand nombre), mathématique (i.e. capacité à observer des éléments saillants dans des structures de nombres sans forcément passer par le calcul), artistique (i.e. capacité à dessiner, sculpter ou jouer d'un instrument de musique), spatiale (i.e. capacité à se repérer dans l'espace sans avoir recours à un outil), kinésique (i.e. capacité à désolidariser ses membres afin de pouvoir faire plusieurs choses en même temps ou pratiquer des exercices physiques 
complexes), naturaliste (i.e. capacité à interpréter des données sensorielles par une perception intelligente, par exemple une carte marine), interpersonnelle (i.e. capacité d'empathie), et intrapersonnelle (i.e. capacité d'introspection). Dans une certaine mesure, ces huit intelligences couvrent un spectre de techniques et d'émotions qui réhabilitent et raccordent intelligence analytique et intelligence émotionnelle. Nonobstant ces considérations, elles représentent un creuset utile à quiconque souhaite multiplier et développer ses compétences, que ce soit dans sa vie personnelle ou dans l'entreprise. Dans ce dernier cas justement, de très nombreuses études empiriques démontrent les effets de l'intelligence individuelle dans le travail et la performance.

Des chercheurs ont constaté que l'intelligence en tant que capacité cognitive générale est un prédicteur important de la performance des tâches, en particulier pour les emplois complexes (GotTfredson, 1986 ; Hunter, 1986). Chatman (1994) associe l'intelligence et un travail acharné pour un impact plus important sur la performance et la réussite professionnelle. De même, Wright, Kacmar, McMahan et Deleeuw (1995) ont de leur côté démontré que les capacités cognitives en interaction avec la personnalité ont eu d'importants effets sur le développement des connaissances liées au travail et à la performance. ReE, CARRETTA et TEACHOUT (1995) ont constaté que les capacités cognitives affectaient directement l'acquisition des connaissances professionnelles et affectaient indirectement les performances du travail. Ainsi, il existe une validation empirique importante sur le rôle de l'intelligence sur le travail et la performance. Malgré ces preuves, la perspective cognitive - ou psychométrique - a été accusée d'être trop limitée car elle n'intègre pas la gamme complète d'activités humaines qui pourraient être qualifiées d'intelligentes ( $c f$. intelligences de Gardner). En outre, il peut s'agir d'une mesure d'intelligence centrée sur l'individu qui ne tient pas compte de la manière dont l'intelligence peut être définie et liée au contexte social ou culturel dans lequel elle se développe et fonctionne (STERNBERG, 1988 ; GARDNER, 1983, 1993 \& 1999). D'autres recherches se focalisent sur les comparaisons entre les individus (i.e. intelligence supérieure vs. inférieure) sur des points comme l'originalité, la préférence pour la complexité, la maîtrise et la flexibilité conceptuelles (STEINER, 1965).

Bien que ces recherches témoignent d'un lien entre l'intelligence et la créativité, des niveaux élevés d'intelligence ne garantissent pas toujours une grande créativité. Amabile (1988) a noté qu'elle n'est pas nécessairement liée à la quantité de connaissances dans un domaine donné, mais plutôt à la manière dont ces connaissances sont stockées et accessibles.

Pour Glynn (1996) l'innovation est impossible en l'absence de génies créatifs capables d'initier des processus innovants et des systèmes organisationnels intelligents. 
Il ressort de tout ceci que l'intelligence est une réalité complexe, source de création de valeur et méritant incontestablement que l'on s'y penche dans une perspective managériale puisqu'elle est au croisement à la fois de la gestion des talents, de la définition des potentiels et des sources traditionnelles de discriminations par la compétence, notamment en termes de diplômes et de formations universitaires. C'est ce que nous allons maintenant discuter.

\section{SYNTHESE SEMANTIQUE \& DISCUSSION}

Talents, hauts potentiels et HQI sont donc des concepts qui trouvent certainement autant de divergences dans leurs acceptions littérales que de convergences dans leurs acceptions managériales. La littérature met clairement en exergue que ces trois formes de ressources humaines sont à la fois espérées et recherchées, voire requises, mais qu'un flou sémantique perdure autour de leurs définitions strictes, sans doute parce que le nombre de variables permettant d'identifier ces sources de performance et de construire des environnements dignes de leur épanouissement est aussi vaste que modulaire.

Ce qui ressort principalement de notre travail, c'est le fait que le HQI qui se positionne par rapport aux notions de talent et de haut potentiel et non l'inverse. Talents et hauts potentiels apparaissent comment étant des extractions de la variable HQI, que ce soit en termes d'acception terminologique ou de méconnaissance des HQI. En effet, le HQI est nécessairement talentueux et bénéficie d'un haut potentiel s'il est identifié et accompagné, alors que le contraire n'est pas vrai ; le talent est unique là où le HQI est pluridisciplinaire et le haut potentiel peut totalement avoir un bel avenir pour des raisons organisationnelles, structurelles, politiques ou contextuelles sans avoir ni une intelligence, ni un talent particulièrement remarquables.

Nous proposons donc le tableau $\mathrm{n}^{\circ} 1$ comme résumé des convergences et des divergences sémantiques tirées de notre analyse de la littérature liant les talents, les potentiels et les HQI.

Ce tableau met notamment en exergue trois réalités managériales.

D'abord, l'entreprise ne valorisera le talent que si ce dernier est en adéquation avec les besoins de la firme, que ces besoins soient récurrents ou sporadiques. Un bon exemple serait de faire appel aux talents de pianiste d'un employé pour une soirée de Noël, compétence inutile le reste de l'année. 
Tableau $\mathbf{n}^{\circ} 1$ : convergences et divergences sémantiques (Source : auteurs).

\begin{tabular}{|l|l|l|l|}
\hline \multicolumn{1}{|c|}{ Caractéristiques } & \multicolumn{1}{|c|}{ TALENTS } & \multicolumn{1}{c|}{ POTENTIELS } & \multicolumn{1}{c|}{ HQI } \\
\hline Individuelles & $\begin{array}{l}\text { Présence de compé- } \\
\text { tences littéralement } \\
\text { "remarquables " }\end{array}$ & $\begin{array}{l}\text { Présence de volonté } \\
\text { et d'envie d'accomplir } \\
\text { pour s'accomplir }\end{array}$ & $\begin{array}{l}\text { Performance homo- } \\
\text { gène sur les 8 formes } \\
\text { d'intelligences de } \\
\text { Gardner (1983, 1993 } \\
\& 1999)\end{array}$ \\
\hline Professionnelles & $\begin{array}{l}\text { Valeur possible mais } \\
\text { pas systématique }\end{array}$ & $\begin{array}{l}\text { Valeur prospective } \\
\text { décidée par l'environ- } \\
\text { nement }\end{array}$ & $\begin{array}{l}\text { Valeur réelle selon } \\
\text { les sensibilités RH } \\
\text { et ouvrant la voie à } \\
\text { la fois à la créativité, } \\
\text { à la rapidité d'ana- } \\
\text { lyse, à la profondeur } \\
\text { de réflexion et à la } \\
\text { résilience en termes } \\
\text { de résolution de pro- } \\
\text { blèmes rencontrés } \\
\text { pour la première fois }\end{array}$ \\
\hline $\begin{array}{l}\text { Gestion Managé- } \\
\text { riale }\end{array}$ & $\begin{array}{l}\text { Valorisation marginale, } \\
\text { c'est-à-dire seule- } \\
\text { ment si le talent est } \\
\text { utile à l'organisation }\end{array}$ & $\begin{array}{l}\text { Valorisation forte car } \\
\text { grandes attentes de la } \\
\text { part du management } \\
\text { et de l'entourage du } \\
\text { sujet }\end{array}$ & $\begin{array}{l}\text { Gestion de la relation } \\
\text { hiérarchique et de } \\
\text { la relation aux pairs } \\
\text { possiblement difficile, } \\
\text { construction d'une re- } \\
\text { lation saine nécessaire, } \\
\text { notamment par le biais } \\
\text { d'une communication } \\
\text { interne adaptée }\end{array}$ \\
\hline
\end{tabular}

Ensuite, si un manager a décelé ou cru déceler un fort potentiel chez un collaborateur, cela reste empreint de la subjectivité la plus totale. En effet, la position de détecteur de potentiels - dans la mesure où talents et potentiels sont déjà distincts dans l'esprit du manager - peut mener à une absence de discernement pour atteindre des objectifs que l'on suppose raisonnables, aucune entreprise n'acceptant de croire qu'elle n'a pas un certain nombre de potentiels dans ses rangs. En outre, identifier un individu à haut potentiel impacte certainement l'évaluation de sa progression, que ce soit en termes d'objectifs ou de durée, car on accepte rarement d'avoir investi sur une personne sans y trouver, à terme, un quelconque bénéfice.

Enfin, la gestion des HQI passe nécessairement par la compréhension de leur structure cognitive, par un échange régulier et marqué entre le manager et le collaborateur, que l'un ou l'autre soit le HQI en question d'ailleurs, et surtout par l'ouverture des pratiques RH de l'organisation à la possibilité soulevée par McClelLand (1973) d'identifier les possibilités d'un collaborateur plutôt que de donner systématiquement la priorité à l'identification des besoins de l'entreprise. 
Dans une certaine mesure, et pour toutes les raisons évoquées dans cet article, les trois notions de talents, potentiels et HQI sont donc relativement assimilées dans l'acception RH, alors que nous pensons qu'elles méritent d'être clairement distinguées. En effet, nous proposons de définir et de clarifier ces notions, socles de nos recherches futures sur les HQI, de la manière suivante :

- En premier lieu, les talents. Nous proposons de définir une personne talentueuse comme ayant des compétences rares. Le Larousse 2017 va d'ailleurs dans ce sens en définissant le talent comme étant « une aptitude particulière ou remarquable à faire quelque chose. »

- En second lieu, les potentiels. De notre point de vue, le potentiel constitue une promesse sur l'avenir, avec tout ce qu'elle comporte d'aléatoire. En définitive, tout individu a un potentiel susceptible de fleurir dans la mesure où il décide de l'utiliser et où l'environnement lui en donne les moyens. Le potentiel est donc impossible à mesurer, impossible à quantifier et, surtout impossible à délimiter puisque n'importe quelle action dépassant une prévision peut donner raison à une prédiction de présence de potentiel. En outre, le potentiel peut très bien être lié à des variables strictement organisationnelles ou relationnelles. Nous constatons ainsi souvent que des entreprises familiales sont cédées par les créateurs à leurs enfants, ces derniers présentant aux yeux du dirigeant un potentiel indiscutable pour continuer à faire prospérer l'entreprise.

- Enfin les HQI. Dans ce cas, nous considérons être en présence d'individus dont les psychologues ont clairement identifié les traits de caractère et dont la force réside soit dans une maîtrise naturelle de toutes les formes d'intelligences identifiées par GARDNER (1983, 1993 \& 1999), soit dans la détention d'une seule intelligence mais atteignant des niveaux prodigieux. On parle alors justement de « prodige » ou de " génie », des personnes dont les capacités de compréhension et de réalisation vont au-delà de toutes les moyennes, que ce soit en termes de diversité d'action, de vitesse d'assimilation ou de précision. A titre d'exemple, nous pourrions citer les très jeunes enfants capables de jouer d'un instrument de musique avec la virtuosité d'un maître, les joueurs d'échecs capables de disputer plusieurs parties en même temps tout en anticipant des dizaines de coups d'avance, ou encore les calculateurs prodiges capables de réaliser des prouesses mentales - par exemple l'extraction d'une racine treizième d'un nombre constitué de 200 chiffres ou encore la mémorisation de dizaines de milliers de décimales de $\mathrm{Pi}$ - encore une fois en empruntant des voies cognitives différentes, voies dont la synesthésie est l'une des manifestations les plus prégnantes. Lorsque l'intelligence est homogène sur toutes les formes d'intelligence de GARDNER (1983, 1993 \& 1999), le sujet est normalement en mesure de créer de la valeur grâce aux quatre caractéristiques qui sont récurrentes chez la plupart des sujets HQI, c'est-à-dire qui contribuent collégialement à gagner du temps, tout en augmentant la qualité et la performance de la ressource humaine : 
- Une créativité inattendue car souvent transversale : par exemple, le sujet HQI pourra associer une faculté d'intelligence spatiale pour résoudre un problème mathématique.

- Une réflexion profonde : le sujet poussera une analyse à son maximum théorique.

- Une analyse rapide : le sujet trouvera une solution à un problème à partir d'un volume d'information très inférieur à ce que l'individu tout-venant utiliserait.

- Une grande résilience : du fait de sa performance sur l'ensemble des intelligences de GARDNER (1983, 1993 \& 1999) et surtout à sa capacité naturelle à interconnecter ces outils intellectuels, le sujet trouvera une solution souvent optimale à un problème rencontré pour la première fois. Le pendant de ce dernier point étant que la complexité de sa structure cognitive oblige quasi nécessairement le problème à être lui-même complexe. En effet, face à un défi simple, le sujet HQI peut devenir totalement désemparé et incapable d'agir, justement parce qu'à ses yeux, sans complexité le problème n'a pas de raison d'être.

Par conséquent, à l'issue de cette revue de littérature et de cette analyse, nous sommes en mesure d'affirmer que :

- les talents, les potentiels et les HQI sont des concepts différents qui, s'ils accueillent certaines convergences perceptuelles, n'en demeurent pas moins éminemment distincts ;

- un individu peut avoir un talent sans être HQI et sans que ce talent représente un potentiel pour l'organisation ;

- un individu peut avoir un potentiel pour l'organisation, sans que ce potentiel soit lié de près ou de loin avec un talent ou un HQI ;

- un individu HQI a automatiquement de multiples talents mais ne représente pas systématiquement un potentiel pour l'organisation. Pour que cela soit le cas, il faut que cette dernière l'identifie comme tel, lui procure un environnement de travail particulier et, bien entendu, que l'activité de l'organisation ait le « potentiel » d'optimiser au sein du collectif de travail les «talents » du HQI, notamment en lui offrant une vision prospective de sa propre croissance, donc un accompagnement.

\section{CONCLUSION}

Outre la crainte d'en considérer la méthode comme une forme de discrimination, si les pratiques RH ne mesurent pas directement l'intelligence, c'est souvent parce qu'elles agrègent les trois concepts que sont les HQI, les Hauts Potentiels et les Talents. En ce qui nous concerne, considérer l'intelligence comme une variable distincte nous apparait pertinent, notamment en termes de gestion de la diversité, de remise en question des pratiques RH et surtout d'optimisation des bénéfices liés à l'existence de structures cognitives différentes. A ce titre, l'objectif de cet article était de questionner les acceptions managériales des concepts de HQI, de Hauts Potentiels et de Talents, afin 
d'en identifier les éventuels points de convergence et de divergence permettant de clarifier à la fois leur étendue sémantique et leurs conséquences en termes de GRH.

Notre recherche a identifié les individus talentueux comme étant des personnes porteuses de compétences rares, les personnes à haut potentiel comme étant des individus faisant partie d'un ensemble de ressources qui, raisonnablement gérées, pouvaient s'avérer prometteuses en termes de résultats futurs, et les HQI comme étant des phénomènes intellectuels particulièrement surprenants, souvent incompris, la plupart du temps découverts par sérendipité et rarement considérés comme sources de création de valeur stable et pérenne.

En ce qui concerne justement le cas des individus HQI, la moitié des intelligences multiples qu'Howard Gardner a identifiées dès 1983 mériteraient sans doute déjà d'être mesurées, dans le cadre des protocoles RH visant à détecter potentiels et talents. Nous pensons notamment ici aux intelligences verbale (i.e. capacité à exprimer des idées de façon claire et intelligible du plus grand nombre), mathématique (i.e. capacité à observer des éléments saillants dans des structures de nombres sans forcément passer par le calcul), naturaliste (i.e. capacité à interpréter des données sensorielles par une perception intelligente, par exemple une carte marine), et interpersonnelle (i.e. capacité d'empathie). Nonobstant l'exigence d'accompagner l'individu doué de ces formes d'intelligences, l'organisation pourrait bénéficier de ressources cognitives individuelles particulièrement performantes, notamment en termes de créativité, de rapidité d'analyse, de profondeur de réflexion et de résilience face à la gestion de situations nouvellement rencontrées.

En parallèle de ces résultats encourageants, la principale limite de ce travail réside dans l'absence de comparaison sémantique entre plusieurs langues étrangères, ainsi qu'entre les cultures qui leur sont associées. En effet, outre les différences de langage entre, par exemple, le terme de "surdoué » pour les canadiens francophones et celui de « gifted » pour les anglo-saxons, il serait intéressant de considérer l'influence des prismes culturels. Par exemple, les États-Unis ont une longue tradition d'identification des HQI, alors que la France est encore timide dans cette démarche une fois l'individu sorti de l'enfance.

Mais s'il ne fait aucun doute pour nous que talents, potentiels et HQI sont des réalités humaines dont tous les managers devraient considérer l'existence et le rôle, les HQI se distinguent néanmoins facilement des deux autres concepts, notamment par leur propension à agréger les trois variables que sont l'intelligence, la compétence rare et la promesse de performance à venir. L'évaluation de la performance individuelle reste encore trop souvent intriquée à celle de la performance structurelle ou de projet, laissant ainsi peu de place à la détection de particularités comme les HQI. 
Quoi qu'il en soit, dans le cadre de nos recherches, un paradigme reste dominant : nous pensons que les pratiques RH mériteraient d'enrichir leurs indicateurs de performance avec des indicateurs d'intelligence, afin que cette dernière puisse être considérée comme un réel facteur de succès des organisations, facteur exigeant donc également une ouverture des GRH à l'accompagnement des individus HQI. La réalité d'entreprises telles que Saint-Gobain, précurseurs en matière de gestion des talents en France, nous prouve que cette idée fait déjà son chemin. L'évolution des métiers et des connaissances étant de plus en plus rapide, il nous semble plus que jamais urgent d'inviter les professionnels RH à (re)considérer la valeur d'une ressource humaine en priorité sur la base de sa propension à se montrer intellectuellement profondément créative et rapidement résiliente.

\section{BIBLIOGRAPHIE}

(de) Ajuriaguerra, J., (1959). Manuel de psychiatrie de l'enfant. Paris : Masson.

Al Ariss, A., Cascio, W.F., \& PaAuwe, J. (2014). Talent Management: Current Theories and Future Research Directions. Journal of World Business, 49(2), 173-179.

Amabile, T.M. (1988). A Model of Creativity and Innovation in Organizations. In B.M. Staw \& L. L. Cummings (Eds.), Research in Organizational Behavior, 10, 123-167. Greenwich, CT: JAI Press. Besseyre des Horts, C.H. (2011). RH au quotidien. Paris : Dunod.

Boudreau, J.W., \& Ramstad, P. (2005). Talentship and the Evolution of Human Resource Management: From Professional Practices to Strategic Talent Decision Science. Human Resource Planning Journal, 28(2), 17-26.

Bourdieu, P. (1980). La distinction, critique sociale du jugement. Ed. de Minuit, Paris.

BoxalL, P.F. (1992). Strategic Human Resource Management: Beginnings of a New Theoretical Sophistication. Human Resource Management Journal, 2(3), 61-79.

Boxall, P.F., \& Purcell, J. (2003). Strategy and Human Resource Management. London: Palgrave Macmillan.

Boyatzis, R. (2008). Competencies in the $21^{\text {st }}$ Century. Journal of Management Development, 27(1), 5-12.

Buckingham, M., Vosburgh, R.M. (2001). The 21st Century Human Resources Function: It's the Talent, Stupid! Human Resource Planning, 24(4), 17-23.

Cappelli, P., \& Keller, J. (2014). Talent Management: Conceptual Approaches and Practical Challenges. Annual Review of Organizational Psychology and Organizational Behavior, 1(1), 305-331.

Chapelier, E ; Sartori, D., \& Schmidt, G. (1999). Détection, évaluation, gestion du potentiel des cadres et des cadres à potentiel. In P. Gilbert et G. Schmidt, Évaluations des compétences et situations de gestion. Paris : Economica.

Chouhan, V.S., \& Srivastava, S. (2014). Understanding Competencies and Competency Modeling - A Literature Survey. Journal of Business and Management, 16(1), 14-22. 
Collings, D.G., \& Mellahi, K. (2009). Strategic Talent Management: A Review and Research Agenda, Human Resource Management Review, 19, 304-313.

DeJoux, C. (2014). Management et leadership. Paris : Dunod.

Dimick, D.E., \& Murray, V.V. (1978). Correlates of Substantive Policy Decisions in Organizations: The Case of Human Resource Management. Academy of Management Journal, 21 , 611-623.

Dries, N. (2013). The Psychology of Talent Management: A Review and Research Agenda. Human Resource Management Review, 23(4), 272-285.

Falcoz, C. (2001). Cadres, la grande rupture. Paris : La Découverte.

Fesser, M. \& Pellissier-Tanon, A. (2007). Les Hauts Potentiels ? Quelles qualités pour les dirigeants de demain? Paris : Éditions d'Organisation.

Fourmy, M. (2011). Ressources humaines-Stratégie et création de valeur. Vers une économie du capital humain : Vers une économie du capital humain. Maxima Laurent du Mesnil éditeur.

Gallardo-Gallardo, E., Dries, N., \& Gonzalez-Cruz, T. (2012). What is Meant by 'Talent' in Business? Workshop on Talent Management, EIASM, Bruxelles.

Gardner, H. (1983). Frames of Mind: The Theory of Multiple Intelligences. Basic Books-Wiley, New York.

GArdner, H. (1993). Frames of Mind. New York: Basic Books.

GARDNER, H. (1999). Intelligence Reframed: Multiple Intelligences for the 21-Century. Basic Books-Wiley, New York.

Gardner, T.M., \& Wright, P.M. (2009). Implicit Human Resource Management Theory: A Potential Threat to the Internal Validity of Human Resource Practice Measures. The International Journal of Human Resource Management, 20(1), 57-74.

Garrow, V., \& Hirsch, W. (2008). Talent management: Issues of Focus and Fit. Public Personnel Management, 37(4), 389-402.

GlynN, M.A., (1996). Innovative Genius: A Framework for a Relating Individual and Organizational Intelligences to Innovation. Academy of Management Review, 21(4), 1081-1111.

Gould, S.J. (1981). The Mismeasure of Man. W.W. Norton \& Company.

Guignard, J.H., \& Zenasni, F.F. (2004). Les caractéristiques émotionnelles des enfants à haut potentiel. Revue Psychologie Française, 49, 305-319.

Irwin, P. (2008). Competencies and Employer Engagement. Asia Pacific Education Review, 9(1), 63-69.

Janand, A., Guettiche, F., \& Clö̈t, H. (2016). Le management « européen » des talents : au-delà des typologies existantes. Revue de gestion des ressources humaines, 1(99), 45-61.

Lang, G. (2012). Is There Potential in Potentiality? Philosophical Papers, 41(1), 129-147.

Le Boterf, G. (2004). Ingénierie et évaluation des compétences. 6ème édition, Eyrolles, Paris.

Legge, K. (1978). Power, Innovation, and Problem-Solving in Personnel Management. London: McGraw-Hill.

Legge, K. (1989). Human Resource Management: a critical analysis. In (ed) J. Storey, New Perspectives in Human Resource Management, Routledge, London. 
Lemoine, G. (2003). Recruter autrement. Le recrutement par simulation. Paris : Éditions l'Harmattan.

LEPAK, D.P., \& SNELl, S.A. (1999), The Human Resource Architecture: Toward a Theory of Human Capital Allocation and Development. Academy of Management Review, 24(1), 31-48.

LÉvy-Leboyer, C. (2009). La gestion des compétences. Paris : Editions d'Organisation.

Mahoney, T.A., \& Deckop, J.R. (1986). Evolution of Concept and Practice in Personnel Administration/Human Resource Management (PA/HRM). Journal of Management, 12(2), 223-241.

Malik, A.R., \& Singh, P. (2014). 'High Potential' Programs: Let's Hear it for 'B' Players. Human Resource Management Review, 24(4), 330-346.

Malik, N. (2009). Emergence of Strategic Human Resource Management Historical Perspective. Academic Leadership: The Online Journal, 7(1), Article 16.

Marin-Garcia, J.A., \& Tomas, J.M. (2016). Deconstructing AMO Framework: A Systematic Review. Intangible Capital, 12(4), 1040-1087.

Martin, J. (2018). Profiting from multiple intelligences in the workplace. Routledge.

McClelland, D.C. (1973). Testing for competence rather than for intelligence. American Psychologist, 28(1), 1-14.

Michaels, E., Handfield-Jones, H., \& Axelrod, B. (2001). The War for Talent. Boston: Harvard Business Review Press.

Minbaeva, D.\& Collings, D. (2013). Seven Myths of Global Talent Management. The International Journal of Human Resource Management, 24(9), 1762-76.

Mintzberg, H. (2005). Des managers, des vrais, pas des MBA. Paris : Éditions d'Organisation.

Mouillot, Ph., Rouby, E., \& Sainty, F. (2000). Repérage sémantique du concept de compétence dans les domaines de la sociologie, de l'économie et de la gestion. Working paper Économies \& Société.

Mouillot, Ph. (2013). A Marketing Contribution to Knowledge-Based Economies. International Journal of Science, Commerce and Humanities, 1(4), 31-38.

Mirallès, P. (2007). La gestion des talents : émergence d'un nouveau modèle de management? Management et Avenir, 11, janvier, 29-42.

Naulleau, M. (2015). Élaboration d'une stratégie de management du talent : quelques conditions de réussite illustrées par une recherche-action, 18(3), Finance Contrôle Stratégie, 18(3), [En ligne].

Naqvi, F. (2009). Competency Mapping and Managing Talent. ICAI Journal of Management Research, 8(1), 85-94.

Nisbett, R.E., Aronson, J., Blair, C., Dickens, W., Flynn, J.R., Halpern, D.F., \& TurKHEIMER, E. (2012). Intelligence. New Findings and Theoretical Developments. American Psychologist, 67(2), 130-159.

Palmer, G. (1988). Human Resource Management and Organizational Analysis. In G. Palmer (Ed.), Australian Personnel Management; A Reader, (pp. 148-163). Melbourne: Macmillan. Penrose, E.T. (1959). The Theory of the Growth of the Firm. New York: John Wiley.

Perrenoud, P. (2018). Construire des compétences dès l'école. Ed. ESF. 
Peretti, J.M. (2009). Tous talentueux : Développer les talents et les potentiels dans l'entreprise, Paris : Eyrolles, Éditions d'Organisation.

Peretti, J. M. (2018). Gestion des ressources humaines. Vuibert.

Ree, M.I., Carretta, T.R., \& Teachaut, M.S. (1995). Role of Ability and Prior Job Knowledge in Complex Training Performance. Journal of Applied Psychology, 80, 721-730.

Roger, A. \& Tremblay, M. (1992). La gestion de la relève dans les entreprises. Revue de Gestion, 17(3), 64-72.

Roussillon, S. (2006). La gestion des cadres à potentiel. In J. Allouche, Encyclopédie des Ressources Humaines, 67-77.

Saint-Giniez (de), V. \& Bernard, A. (2000). Les jugements d'attribution du potentiel, une approche constructiviste. In F. Bournois et S. Roussillon, Préparer les dirigeants de demain. Paris : Editions d'Organisation.

SAmElson, F. (1982). Intelligence and Some of its Testers. Science, 215, 656-657.

ScouArnec, A. (2010). Management et métier - Visions d'experts. Paris : Editions EMS.

Sisson, K. (1993). In Search of HRM. British Journal of Industrial Relations, 31(2), 201-210.

SPEARman, C. (1904). "General Intelligence”, Objectively Determined and Measured. American Journal of Psychology, 15, 201-293.

SPEncer, L., \& SPencer, S. (1993). Competence at Work: Model for Superior Performance. John Wiley \& Sons, New York.

Steiner, G. (1965). The Creative Organization. Chicago: University of Chicago Press.

Sternberg, R.J. (1988). A Three-Facet Model of Creativity. In R.J. Sternberg (Ed.), The Nature of Creativity: Contemporary Psychological Views, 125-147. Cambridge, England: Cambridge University Press.

Thévenet, M. (2012). Gérer les talents. In M. Thévenet, C. Dejoux, E. Marbot, E. Normand, A-F. Bender, \& F. Silva, Fonctions RH : politiques, métiers et outils des ressources humaines. Paris : Pearson.

Thévenet, M., \& DeJoux, C. (2010). La gestion des talents : La GRH d'après-crise. Management Sup, Dunod ed.

Thunnissen, M., Boselie, P., \& Fruytier, B. (2013). A Review of Talent Management: "Infancy or Adolescence"? The International Journal of Human Resource Management, 24(9), 17441761.

Truss, C., \& Gratton, L. (1994). Strategic Human Resource Management: A Conceptual Approach. 5(3), 663-686.

Ulrich, D., \& Smallwood, N. (2012). What is talent? Leader to Leader, 63, 55-61.

Vaiman, V., \& Collings, D.G. (2013). Talent Management: Advancing the Field. The International Journal of Human Resource Management, 24(9), 1737-1743.

Watzlawick, P. (1964). An Anthology of Human Communication, Text and Tape. Science and Behavior Book.

Wechsler, D. (1944). The Measurement of Adult Intelligence. New York: The Williams \& Wilkins Company.

Winner, E. (1997). Surdoués. Mythes et Réalités. Paris : Aubier. 